\title{
Quelques réflexions à travers une expérience du CNES en mécanique
}

\author{
GÉrard Jeanblanc ${ }^{1, a}$, François Dugué ${ }^{2}$ et Michel Illig $^{3}$ \\ 1103 rue Brancas, 92310 Sèvres, France \\ 2 CSTM, 35 rue de Toulouse, 31320 Castanet-Tolosan Cedex, France \\ 3 CNES
}

Reçu le 1 juillet 2008, accepté le 17 octobre 2008

\begin{abstract}
Résumé - Ces descriptions schématiques d'actions de Recherche et Technologie, sont situées dans un parcours collatéral en relation avec les débuts des projets Ariane. Elles ont été conduites à l'aide de groupements interdisciplinaires dont les GdR du CNRS, et comportant des acteurs scientifiques, enseignantschercheurs, ingénieurs, technologues et industriels. La démarche technologique et industrielle de construction est présentée à l'aide des fonctions techniques en mécanique. L'objet technique est visé, à partir de conditions initiales des objectifs affichés comme projet, par la mise en œuvre des flux d'énergies, de produits, d'acteurs, et d'informations industrielles. Ceci s'associe à la maîtrise du comportement en utilisation effective et à l'utilisation des connaissances exprimées par la recherche et l'enseignement. On incorpore donc au long du déroulé opératoire des repères, lieu de couplages amont/aval et aval/amont.
\end{abstract}

Mots clés : Recherche / mécanique / objet technique / construction / connaissances / projet

Abstract - A few thoughts around practices linked to CNES about mechanics. Schematic descriptions of actions for Research and Technology are represented in a collateral course related with the beginning of Ariane projects. These have been managed by means of interdisciplinary groups like the GdR of CNRS, including scientifics, teachers, engineers, technologists and manufacturers. The technological and industrial steps, like way of product design, are presented by the technical functions in mechanics. The technical object is aimed, from initial conditions of objectives shown as plan, by implementation of energies, products, actors, industrial informations, starting from objectives designed as project. This design is bound to the control of behavior in operations and to incorporate the knowledges of research and teaching. For what the landmarks on flowchart, gives the opportunity, for, upstream/downstream and downstream/upstream, connections.

Key words: Research / mechanics / technical object / product design / knowledges / project

\section{Introduction}

\section{Passage de l'idée à l'objet}

Ceci repose, pour l'ingénieur en mécanique, entre autres, sur l'insertion de la technologie, dans une organisation de projet. Les auteurs, partageant des expériences communes se proposent de décrire des démarches de travail, avec une intention plus mnémotechnique que prescriptive. L'utilité de l'association entre les compétences des disciplines scientifiques et les connaissances des contextes de développement et d'emploi, a été constatée chez nos prédécesseurs. Ceci a été adapté aux outils et pratiques, dans le domaine des recherches, pilotées par des

\footnotetext{
a Auteur pour correspondance: gerardjeanblanc@orange.fr
}

besoins associés à des systèmes spatiaux. On schématise ceci par les descriptions suivantes portant sur une période de plus d'une décennie, pour susciter l'échange avec les scientifiques générateurs et développeurs des connaissances, ainsi qu'avec les managers confrontés aux contraintes grandissantes du futur, mais surtout avec les jeunes générations.

\section{Une expérience partagée}

Activités coopératives et multidisciplinaires

La réalisation d'objets et l'expression appliquée de théories, utilisant les connaissances scientifiques et technologiques en mécanique, sont ici, pour une bonne part, le fait de coopérations entre enseignants-chercheurs et 
ingénieurs praticiens des objets industriels du domaine spatial.

Utilisées dans le cadre de petites équipes associant des spécialités différentes, ces pratiques découlent de celles des activités spatiales industrielles qui, plus structurées et plus détaillées ont également été transposées dans d'autres domaines.

\subsection{R\&T associée avec Ariane}

\section{Contexte des besoins et pratiques de l'ingénierie}

On s'appuie sur quelques expériences, dans un contexte d'ingénierie, lors d'une période, associée à la réalisation de la filière Ariane 1/5. Celle-ci combinait, dans la direction principale exercée par Frédéric d'Allest [1] et Roger Vignelles, les démarches de développement nord-américaines [2] et des pratiques européennes, essentiellement britanniques et françaises [3]. Les institutionnels et industriels, ont préparé, dès 1976 , les évolutions technologiques conduisant à Ariane 5, selon les capacités d'innovation de recherche utilisées par le CNES pour les satellites scientifiques ou applicatifs, et par l'industrie aérospatiale, en entraînant, des équipes de recherche d'organismes publics, d'entités de formations supérieures, ainsi que des PME. Les premiers tirs d'Ariane 1, avec leur part de défauts de jeunesse, bien connus des adeptes des courbes d'apprentissage fiabiliste, ont conforté, avec la préparation des développements futurs, l'appel aux compétences extérieures aux projets proprement dits.

La répartition des tâches et le caractère restreint des équipes, résultat des décisions du premier Directeur du CNES, Robert Aubinière, rendaient nécessaire l'élargissement des liaisons extérieures. Quelques exemples : en premier lieu, la poursuite des activités antérieures, par l'ONERA sur des thèmes comme le POGO [4], couplage vibratoire entre le jet des gaz propulsifs et les structures, popularisé par un numéro de « la recherche » dans les années 80, s'est enrichi du concours d'équipes du CNRS sur la combustion après les instabilités rencontrées en vol sur LO2, le deuxième lancement d'Ariane.

En sus, des « champs nouveaux» ont été prolongés pour les lanceurs, comme sur les aspects tribologiques en lubrification limite (avec Supmeca, INSA, ENSAM,...$)$ des contacts en milieu de fluides cryotechniques, par la mise en place de moyens d'essais ou la recherche d'expertise. Enfin la préparation du futur a pris en compte la naissance de l'informatique individuelle avec la reprise de méthodes d'itération (réseaux de pertes de charges par ECP et ENSAM/LTCE, méthode des caractéristiques), de celles de l'automatique (ENSAM/CNAM, Simulog), mais aussi de sujets traités anciennement par les méthodes graphiques (dynamique des rotors avec Louradour et Blanc...). La mise en œuvre de méthodologies nouvelles (écoulements rotationnels : LIMSI), et la coopération université-industrie, propre à la région de Grenoble (cavitation), se sont développées, ainsi que l'appel aux compétences de l'INRIA.
Ces équipes ont été confrontées aux aspects contractuels, administratifs et techniques des relations entre industriels et CNES. Tous les participants ont incorporé avec quelques difficultés mais avec humour les processus des marchés publics, situant les rôles respectifs, grâce à l'acquis de participants, acteurs de programmes nationaux de l'aéronautique et de l'énergétique. Les industriels et en particulier ceux de la propulsion ont largement contribué à la participation et au pilotage d'actions technologiques prolongées par des démonstrateurs décrits par Beaurain et Souchier [5].

Le développement du programme Ariane suppose un maintien des activités prioritaires d'équipes à effectif quasi-constant au CNES pour le pilotage et le soutien technique des projets. Ceci entraînait une tension avec la vision prospective qui conduit à élargir le spectre des activités, comme vers les méthodologies [6] de l'informatique avancée, avec le CEA, l'ENS Cachan, le CETIM, entre autres. Les développements technologiques impliquent dans leurs composantes techniques, l'adéquation « fonctionnelle, dimensionnelle et fiabiliste », et sur le plan industriel la prise en compte de la faisabilité, du suivi de la configuration et de la qualité [7] en respectant les contraintes économiques et opérationnelles. Cela est d'autant plus prégnant aujourd'hui avec l'accroissement de la cadence des lancements lourds d'Ariane 5 et les évolutions définies par des études d'avant-projets [8]. De même, les charges des enseignants-chercheurs en termes d'administration et de conduite de leurs entités, ne leur permettaient pas facilement d'en élargir les horizons.

Ces charges, tôt perçues par Hubert Curien et René Pellat en liaison avec les directions des entreprises industrielles, ont conduit vers des regroupements, tout en poursuivant les actions ponctuelles directes du CNES, avec des équipes de recherche ou avec celles des équipes industrielles tournées vers les applications technologiques. Ces associations de différentes équipes en groupe, lourdes sur le plan contractuel, ont eu un effet réciproque de formation indéniable, par la concrétisation des problèmes pratiques et des approches théoriques, pour les connaissances enseignées dans les formations d'ingénieurs et pour l'enrichissement des démarches utilisées par les acteurs industriels.

Ceci s'est inscrit dans des programmes dénommés de «R\&T (Recherche et Technologie)» pour en centrer le management en l'allégeant des activités de développement, qui préfigurent celui de la production et, selon R. Pellat, pour ainsi « mieux... organiser le transfert des compétences... l'utilisation des organismes extérieurs... le regroupement pluridisciplinaire » [9]. Pour certains aspects, un cadre contractuel essentiellement local a été dénommé pôle ou GRT, avec une entité pilote et des règles explicites de coopération entre les ingénieurs du CNES, les responsables des actions, et des équipes de recherche ou des supports techniques. Cette modalité a assuré une cohérence en incluant des PME, en prenant en compte l'excellence, mais aussi la rapidité de liaison, par la proximité. Ce type de travail, calqué sur les modalités des répartitions industrielles du moment, visait plutôt 
la prise en compte des contraintes aval de technologie et d'utilisation. Une personnalité scientifique conduisait une évaluation en liaison avec l'ensemble de celles d'une commission d'évaluation auprès du CNES, permettant la transition vers des activités de recherche plus structurées. Une attention a été portée aux moyens d'essais et logiciels, face à l'évolution des matériels et des systèmes d'exploitation, ainsi qu'aux modalités juridiques de propriété. Cette démarche de regroupement de spécialités tourné vers un objet ou un domaine, s'est élargie, comme maintenant sur le plan régional, par les pôles de compétitivité, mais auparavant par les GdR.

\subsection{Actions dans le cadre de GdR}

\section{Interactions des équipes scientifiques}

L'association de l'excellence des connaissances amont, dans une structure de laboratoire «sans mur », à savoir ici les GdR (groupements de recherche du CNRS/SPI maintenant STII), ont facilité les financements conjoints, l'identification des compétences pour une progression des connaissances et leur insertion dans les processus industriels (investissements, règles internes, brevets...) et scientifiques (bourses, thèses, publications, échanges...).

Cette modalité, moyen terme entre la recherche et les travaux industriels, permet de traiter des questions complexes et de rechercher des solutions stables sur l'ensemble des cycles de vie d'un objet potentiel. Elle a été appliquée à des sujets divers, tels que roulements, combustion, étanchéités, puis propulsion à plasma, selon les axes suivants :

- « regroupement de compétences » régionales ou nationales par une négociation (juridique, tutelle administrative, scientifique et technique) sur la base de référence du GdR CNRS/SPI, d'une convention ou protocole d'accord, entre parties concernées, (avec expression de règles communes quant à la constitution des instances (comité directeur, conseil scientifique et direction de groupement)), avec un document d'organisation précisant les documents associés à une séquence de travail;

- « conduite des actions par les acteurs », après mise en place par les parties : désignation des membres, identification des objectifs dans le champ d'études, dans un cadre quadriennal, programmation des projets, engagement des modélisations et expérimentations, déroulement des projets, identification des résultats;

- « direction par un doublet de personnalités », au fait des aspects applicatifs et théoriques : directeur du groupement en charge de l'enchaînement pratique des actions et projets en relation avec le président d'un conseil scientifique.

Des travaux pluridisciplinaires par les publications et thèses apportent in fine, une meilleure pérennité et réactivité de la conception.

\subsection{Démarche technologique et industrielle}

\author{
Passage de l'idée à l'objet par le projet pour structurer \\ la démarche
}

Revenons au point de vue et à la situation de l'ingénieur. Différentes facettes illustrent le terme de «mécanique », telles que connaissances scientifiques formalisées, applications pratiques et construction d'objets artisanaux ou techniques. Fréquents sont les usages analogiques [10] dans les sciences de la nature et la transposition des vocables, par recherche d'équivalence de modèles par les sciences humaines [11,12] et économiques [13]. Le débat entre la science et l'industrie, avait été posé par Le Chatelier [14] dans des interactions constructives entre la science et l'industrie par une synthèse des méthodes de Descartes, Taylor et Claude Bernard, avec la nécessité des mesures pour l'expérimentation et des contrôles pour une production efficace. Il est relancé en 2008, sur le site AFM, par un diagramme, intitulé « Qu'est-ce que la mécanique ». Il est constitué de réseaux concentriques, depuis les différentes sciences jusqu'aux domaines de l'activité humaine illustrant les champs d'applications sans intermédiaires. Tout cela entoure les pôles de la mécanique de solides et des fluides avec l'exergue « du modèle à l'application ».

Pour relier aux sciences, la démarche présentée, revenons à Gagnepain [15] situant la recherche technologique « entre la recherche d'un savoir et celle d'un bienêtre », qui passe de l'action imaginée à sa concrétisation, ou du besoin vers l'utilisateur selon l'économie [16], c'està-dire de «l'idée vers l'objet [17] » et maintenant vers le système $[18,19]$ pour l'ingénieur. Celui-ci, dépositaire des acquis [6], associe les théories scientifiques, avec par exemple la connaissance pratique des outils [20], interaction mécanique [21] immédiate avec la matière.

Ainsi, les logiciels, terme générique créé par Nora et Minc [22], successeurs des tracés des compagnons charpentiers ou des formes des forgerons [23], sont, dans la conception, les supports fonctionnels d'informations, intermédiaires de fait vers «l'objet technique ». Maintenant, ils sont reprécisés curieusement « software computer » dans l'ISO 9000 (version $2000 \S 342$ nota 4), au prétexte que software est un terme plus générique, alors qu'en fait les sens de logiciel se sont étendus. Au-delà de cette dérive étonnante pour les linguistes, c'est au moins un signe que la technique et la technologie sont liées aux langages. Plus généralement on peut suivre l'objet, innovant, traditionnel, artisanal, prototype ou industriel, dans son élaboration, à travers l'entrelacs des processus avec les étapes de la construction entre :

- connaissances scientifiques formalisées (théories classiques, ou descriptions logiques des systèmes à base de connaissances [24]);

- comportements expérimentaux sous sollicitations nominales ou extrêmes ;

- conduites, au sens des automaticiens, sous perturbations. 


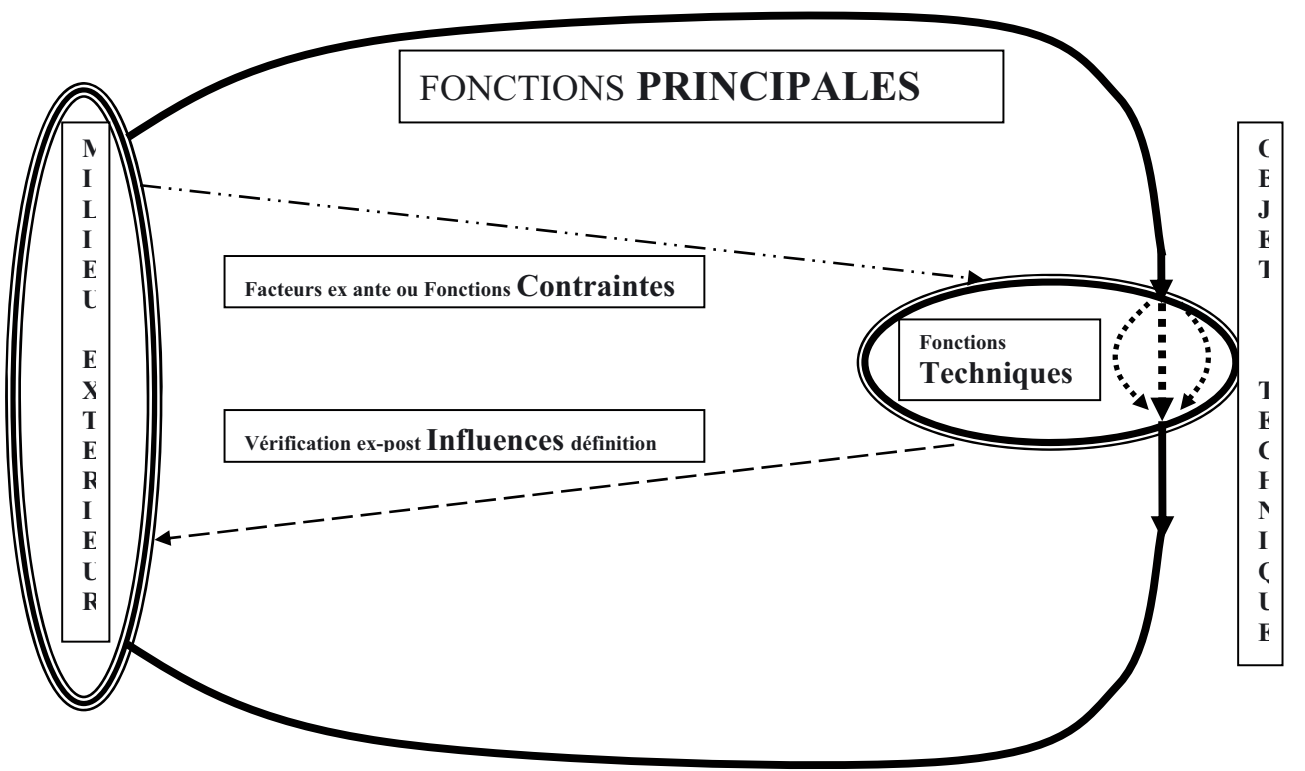

Fig. 1. Relations milieu exterieur et objet technique : fonctions principales, contrainte et influence.

Par rapport aux aspects industriels, ce déroulement schématise, ici, l'échange entre intervenants dans ces processus, en s'appuyant, sur des analogies $[25,26]$, comme celles de la mécanique des fluides ou des réseaux électriques (Forrester [27]). Relevant, de domaines scientifiques, et métiers fort variés, cette représentation des aspects techniques et industriels est une simple ébauche, indispensable aux acteurs et parties prenantes.

Elle concerne néanmoins, aussi, les enseignants et chercheurs qui assurent, par période, tels de modernes Cincinnatus, ces fonctions de construction dans le cadre des responsabilités déléguées de décision, de gestion d'installation et d'équipes associant industriels et chercheurs [28]. D'autant plus que des aspects essentiels de la mécanique, en particulier aux interfaces, à l'exemple de la tribologie, sont, comme le rappelle Jean Frene [29] « complexes et multidisciplinaires (pour des raisons) scientifiques et techniques (mais aussi du fait) des aspects industriels (et opérationnels) ». Passer de l'idée à l'objet implique, en dehors d'un instantané imaginaire, une séquence [30] que l'on peut définir, par des étapes marquées par des jalons, dont le caractère conventionnel est propre à chaque organisation [30]. Par exemple on peut enchaîner depuis le début d'une opération : expression du besoin, conception (et définition), réalisation (avec contrôle), fonctionnement (en essai et en utilisation).

Cette description et donc le passage par les langages [31] assurant le transfert de la pensée au discours [32] pour l'échange, se poursuit selon un processus. Ceci devient support [33] d'un avant et d'un après, porté ici par les « outils» proposés. Ce schéma rationnel de construction transmissible à de petites entités, comporte des étapes utilisées en pratique. Là encore, il faut s'appuyer sur le rappel de Jean Frene [29], directeur de GdR (roulement en milieu cryotechnique puis étanchéité en milieu extrême), à savoir que le développement d'une discipline passe du « descriptif au formel en passant par le phénoménologique ». Ici, cette démarche usuelle est explicitée en s'appuyant sur des analogies, en tant que premier incrément.

\section{Une démarche de construction}

Mise en relation de l'objet potentiel avec son milieu environnant

Le développement de prototypes [34] avancés et de banc d'essais [35] rejoint, selon une vision de système [36], les concepts établis dans le cadre de projets institutionnels ou industriels [37] plus orientés vers la réponse à un usage pratique que vers la connaissance.

On schématise cette base commune de la distinction du nouvel objet inséré dans le contexte ou monde environnant, dans ce qui est qualifié de milieu extérieur, comme en thermodynamique et en biologie. L'objet et le milieu sont en relation, en particulier par une boucle principale représentant l'usage attendu et par de simples injections représentant des contraintes univoques.

Ces relations, issues des travaux scientifiques et des connaissances techniques, ont été propagées par les enseignements de L. Géminard, sur les itérations de la conception en mécanique [38] et la liaison des fonctions techniques [39] avec l'analyse de la valeur [40]. La pédagogie [41] des technologies industrielles dissocie cette démarche, en analyse extérieure des besoins et sollicitations puis en celle des fonctions intérieures.

Ceci peut devenir en partie contradictoire avec la pratique du juste nécessaire et de la vérification de la robustesse. Une perte du sens courant [42] conduit hors de l'objectif du fait de l'introduction excessive d'une multiplicité difficile à maitriser. Cela rejoint curieusement le reproche des praticiens de l'analyse de la valeur [40], à l'égard de la surabondance des constituants 
dans les anciennes solutions en conception industrielle. Les connaissances générées par la recherche scientifique et les développements technologiques et structurées pour la communication (publications et enseignement), sont utilisées à travers des actions et moyens (matériels, méthodes) par des acteurs pour construire un objet, un outil, un ensemble (dispositif, machine, moyen d'essai...). L'intégration [18] de matériels et logiciels [43], associant des techniques issues d'autres domaines industriels et scientifiques, conduit à des systèmes $[29,44]$ (véhicule, soutien, ouvrages et servitudes, système à finalités étendues [45], et même une notion préemptée par les linguistes [33] « système de système » (supersystem pour la NASA [30]) parfois source de complexité risquée [46], malgré les techniques d'évitement comportemental [47]...).

L'objet est constitué à partir des flux de ressources, selon un processus itératif à partir des fonctions principales [39]. Les approximations trop larges lors de la conception inévitables en temps limité et en situation complexe, voire les absences ou insuffisances de soutien théorique ou empirique sont sources d'erreurs de même qu'ensuite les écarts de sollicitation et de réalisation, à l'égard desquels on se prémunit par les facteurs et marges de sécurités.

Les grands projets structurés débutent selon une séquence formalisée, à partir d'un contrat ou marché, partant d'un cahier des charges puis servant à élaborer une spécification technique, ici confondues. L'objet est défini en conception pour répondre aux fonctions principales et construit par une réalisation en combinant des fonctions techniques en respectant les liaisons univoques en particulier les fonctions contraintes et en minimisant les influences ou impacts [48] sur le milieu extérieur. Ces dernières peuvent difficilement être traitées comme des contraintes classiques, car peu indentifiables a priori au stade de l'expression du besoin. Par contre elles sont vérifiables comme respectées en conception à partir des supports de définition détaillée, c'est-à-dire en terme courant à partir des dessins et nomenclatures.

\subsection{Système des fonctions techniques}

\section{Regroupement des schémas techniques pour construire}

En utilisant un langage emprunté à l'automatique [49], on peut dire que le passage à la pratique, se fait de façon incrémentale ou en escalier plus que par échelon communément qualifié de « tout ou rien ». Les intermédiaires actifs sont alors constitués par les rapports et revues techniques, appuyées sur les « fonctions techniques combinées ». Cette proposition utilisée dans un cadre de R\&T [50] regroupe les fonctions de base conventionnelles, selon une structure [51] inspirée de l'ingénierie des systèmes :

- Définir les « références », internes et externes, de l'objet, par les fonctions techniques de base :

- définir les « situations relatives » : (phases/corps)

- solide/solide $=$ mise en position ;
- fluide/solide $=$ forme, contenance ou déviation...;

- fluide/fluide = interfaces et phases des volumes ;

- répondre aux contraintes «d'utilisation et d'intégration » dans le système :

- dimensionner (géométrie, choix et marge des matériaux) selon sollicitations volumiques nominales et extrêmes (torseurs aux appuis, charges rotatives, charges réparties par pression...);

- dimensionner aux sollicitations d'endurance (facteurs d'abattement : fatigue, fissuration, fluage...) globales ou ponctuelles, linéiques (cf. René Gras, Tribologie Dunod 2008);

- vérification aux sollicitations surfaciques (tribologiques, agressions physico-chimiques, effets électriques (pelliculaire...), magnétiques (hystérésis,...);

- dimensionner pour les épreuves conventionnelles mécaniques et thermiques ;

- identifier les couplages mécaniques, physiques, électriques (IEM)...;

- maintenir la «stabilité » des situations en regard des sollicitations et perturbations (mécaniques, thermiques, électriques,...);

- objet/système $=$ stabilité statique ;

- solide/solide $=$ mise en position, éloignement des fréquences propres, identification des conditions d'instabilités ;

- surfaces solides $=$ plages utiles des résistances passives adéquates ;

- fluide/solide = débit/perte de charge, flux de fuite...;

- fluide/fluide $=$ stabilité des domaines ;

- définir et dimensionner « fonctionnellement et structurellement $»$ les transferts, transformations et couplages (fonctions techniques combinées, avec potentiels de croissance) et maintenir par ce dimensionnement le contrôle passif des situations et actif de la stabilité en regard des sollicitations et perturbations :

- «transitions » : puissance/énergie sous différentes formes ;

- « situations » : position, mouvement (déformations, déplacement), sens, informations ;

- localisations des « signaux » et caractéristiques des «bouclages » proactifs et rétroactifs;

- identification des « effets $»$ des sollicitations et perturbations sur les:

- caractéristiques internes (dimensionnelles (forme et surface), compositions évolutives);

- entrées de l'extérieur (stabilité dynamique et thermique, résistances (caractéristiques et dimensionnement) aux interactions (physiques, physico-chimiques, électriques...), comportements avec leurs limites indicatrices de sources de défaillances [52])).

On doit noter la nécessité de pratique de vérifications complémentaires telles celle des barrières pour répondre aux exigences apparemment paradoxales comme celle des anciens formateurs des Glénans - indiquant que « si une erreur est possible, elle a déjà été faite, et que sinon elle se 
Tableau 1. Schéma indicatif mécanique d'une arborescence constitutive d'un système.

\begin{tabular}{ccccc}
\hline À qui & Pourquoi & Entité/objet & Fonctions & Sur quoi \\
\hline Charge utile & Énergies & Système & Transfert & Mouvement \\
Lanceur & Résistances $(g, \rho S V, f)$ & Moteur & Poussée & Éjection masse \\
Capacité combustion & Alimentation & Machine tournante & Élever pression & Compressibilité \\
Mouvement fluide & Mouvement (Euler) & Sous-ensemble mécanique & Positions respectives & Rotation \\
Position pièces & Torseur effort & Composants (corps, surface) & Précision et minimiser pertes & Interfaces \\
\hline
\end{tabular}

fera un jour »- ou aussi celle du test de l'erreur inéluctable dite « Murphy proof » visant à poser des détrompages incontournables. C'est le domaine de la sureté de fonctionnement.

Ce tableau est à adapter selon les domaines d'emploi, car les fonctions principales, au sens de l'analyse de la valeur, sont essentiellement celles, porteuses des transferts ou transformations, recherchées.

Un processus pratique a été proposé, par la société APTE (devenu TDC Knowllence), par des questions types formulées [47], comme suit : à qui cela rend-il service (entité/objet), sur quoi cela agit-il et pourquoi cette fonction est-elle nécessaire? On peut prolonger cette interrogation, selon la pratique d'un des concepteurs du lanceur LIIIS préfigurant Ariane 1, en enchaînant des pourquoi du pourquoi.

Cela conduit en passant par des étapes constructives à divers résultats : une loi scientifique ou pour les choix de technologie, par la définition, à des références conventionnelles éventuellement préférentielles (classes de Renard, normes ABEC, ISO, liste de composants préférentiels COTS [53]...). Ce mélange est indiqué dans le tableau 1

Cette analyse fonctionnelle est à utiliser en parallèle avec les démarches usuelles de conception/définition, voire de vérification. Celle-ci fait l'objet de tableaux spécifiques (AMDEC, analyse de risques...) pour les systèmes dans la démarche des fiabilistes.

\subsection{Conditions initiales et flux}

Entrelacs des moyens initiaux et des démarches tournés vers les objectifs

Dans la séquence des questions (QQOQC dites [40] de Taylor, utilisées depuis Quintilien), se pose celle du « comment ». L'emprunt analogique aux domaines mécaniques introduit naturellement les conditions initiales et les flux. En contrepoint, il est utile de regrouper les éléments du management [54] (gestion industrielle, financière, comptable : matières, énergies, informations, finances), et des sciences humaines (stratégie [55], psychologies, cultures, langages...). On assemble, ici, ces flux en quatre agrégats (énergies, produits, acteurs, signaux) en prolongeant les disciplines de l'organisation [27], par les méthodes de description des systèmes [56] :

- transitants : Énergies et Produits (incluant matières, demi-produits, COTS [53]);
- transformants : organisations, outils, machines et organes (signification restreinte à équipements) de transformations, acteurs (parties prenantes, commanditaires et évaluateurs associés selon René Pellat Pdt. du CNES) par extension = Acteurs ;

- informatifs : informatique, financier, comptable, psychosociologiques et leurs supports à travers langages et parcours jalonnés de symboles, signes = Signaux situés dans des documents d'accompagnement procéduraux ou organisationnels (cf. Planchette Nicolet, Valancoque, «Et si les risques m'étaient comptés », Octares éditeur, 2003) et inclus maintenant dans les logiciels d'applications, base de données et des connaissances.

Les passages à la constitution d'un objet se construisent avec les ressources, insérées par les décisions dans les processus, qui sont déclenchés (« kick-off ») aux conditions initiales. À ces instants, on englobe les démarches de management de projet, de qualité et de technique, élargies ici (mnémotechnique ICAM : intentions, connaissances, antériorités, moyens) :

- « intentions » : en élargissant la notion de besoins ou d'objectifs qui ne sont pas toujours exprimables de façon explicite sans dialogue avec les autres parties prenantes ;

- « connaissances » : emprunt à la formalisation [57] préalable au codage en langages déclaratifs, recouvre savoirs scientifiques et agrégats empiriques des domaines physiques et humains;

- « antériorités » : extension de la mémoire technique [58], gestion des connaissances, à partir de l'assurance qualité (insatisfactions, écarts, incidents, dérogations...)

- « moyens »: (ressources et) outils matériels et financiers, méthodes industrielles et opérationnelles.

Ensuite à partir des conditions initiales, les projections se déroulent en associant l'utilisation des moyens et des flux et leur commande avec des contrôles par corrections des écarts, en retour d'expérience ou, à plus long terme, par la mémoire industrielle ou technique. Les processus ajustent les caractéristiques et performances visées dans le cadre des valeurs tolérancées, dont le dépassement entraîne ces actes d'information et de décision [58] réglage, palliatif (mis en exergue par ADEQUA à partir des méthodes de l'École de Palo Alto [47] - correctif, préventif).

Ces éléments sont combinés dans les processus de développement. On propose de préciser et de dissocier les couplages, pour identifier les repères, afin de détailler le déroulé opératoire. 


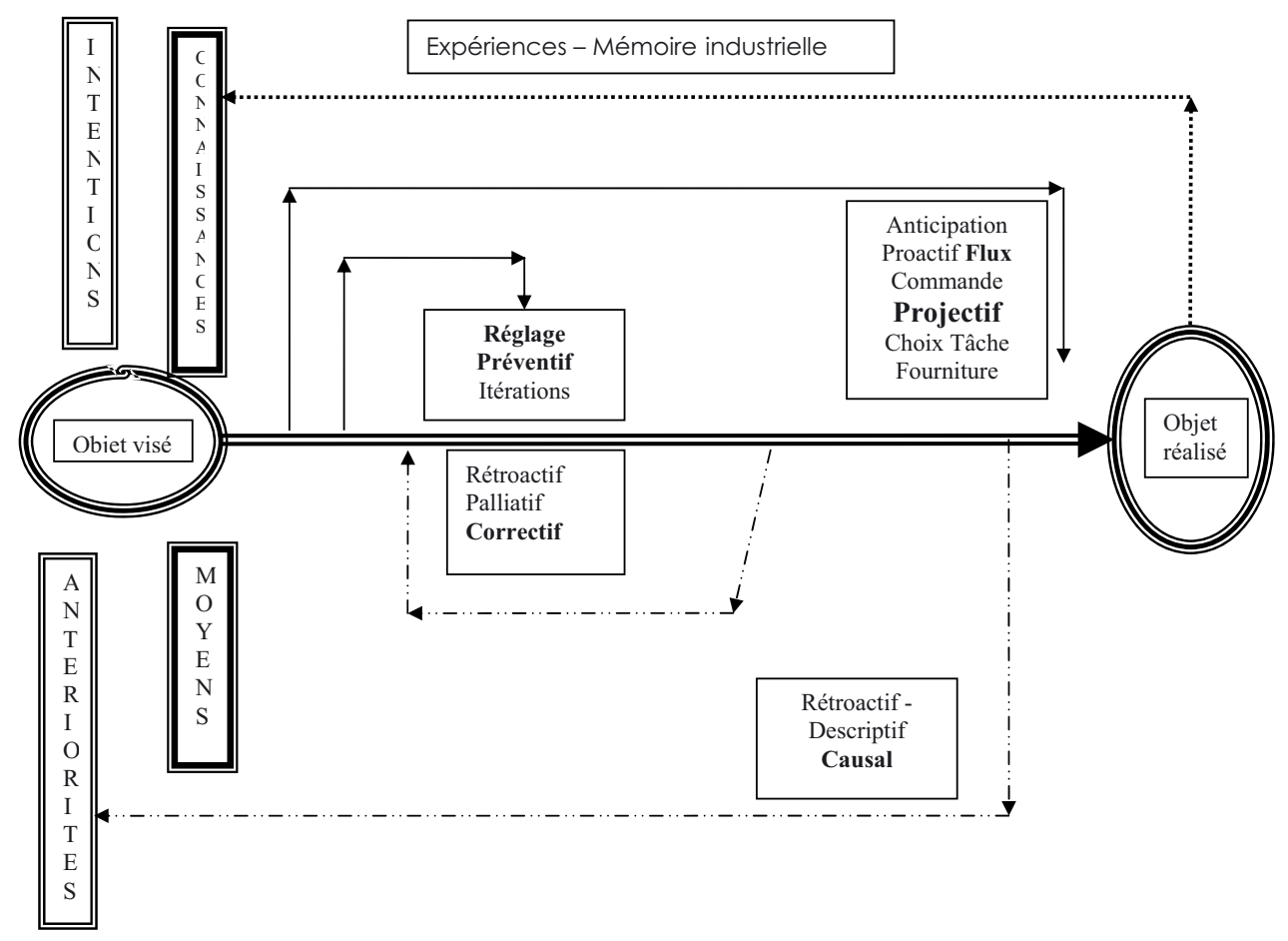

Fig. 2. Conduite : flux EPAS et conditions initiales ICAM.

\subsection{Couplages et repères}

\section{Se situer pour prévoir et faire converger}

Préciser et dissocier les couplages peut se faire essentiellement en répondant à la question suivante, celle du «quand ». Du fait des activités en parallèle, elle est plutôt à reformuler en «avant quoi » ou «après quoi ». Cela est valable pour les jalons de vérification de l'écoulement des flux, et des états de leur transformation. Mais il faut aussi situer les interventions de vérification, par comparaison aux structures de travail définies initialement ou attendues en situations intermédiaires ou terminales. Ceci implique une référence de comparaison, une mesure de l'écart et la mise en œuvre d'ajustements sur les causes potentielles.

Ce sont donc deux flux couplés avec le flux principal de construction : selon l'analogie avec les méthodes de l'automatique en régime harmonique, l'un en projection (avance de phase ou anticipation), le second en retour vers l'amont, ou en adaptations rétroactives, selon la notion ancienne des automaticiens, inconnue de quelques adeptes $\mathrm{du}$ vocable « feed-back ». Dans certaines industries audelà des actions correctives (cf. Fig. 2), c'est une voie d'amélioration par collecte à travers une boîte à idées, dépouillées par les bureaux des méthodes en assurant la retombée des bénéfices identifiés.

Ces couplages sont qualifiables en terme mécanique de butée et de renvoi. Plus généralement, ils sont décrits, dès les ouvrages de technologie de quatrième, en termes de bouclages par ajustement au juste nécessaire, à partir des conditions initiales, en transformant les éléments des flux dans les états intermédiaires vers les structurations projetées. L'instantanéité, utopie motivante pour les commanditaires et gestionnaires, est parfois associée à l'ingénierie simultanée [59] qui vise une préparation sans faille de la fabrication, en utilisant des bases de données de grande ampleur [60], dans un contexte très stable. Mais l'instant implique au moins un avant et un après, séparant l'idée de l'objet. Il y a, en général, nécessité d'itération et donc décalage temporel. Ceci au moins du fait de l'aménagement géométrique (encombrement, architecture...) et du dimensionnement fonctionnel. C'est le cas même dans des processus assez intégrés, avec utilisation de la stéréolithographie pour une pièce. Sont essentielles [61], en mécanique, la tenue de la structure en contrainte/résistance, mais aussi en rigidité et stabilité sans oublier les conditions de la cinématique et de la dynamique des constituants et les conditions d'usage, tel que l'esthétique, aussi, critère de jugement.

Ceci résulte d'associations en coopération, de règles et d'expériences en développement de systèmes complexes. Sans détailler la séquence, on peut déjà regrouper les éléments, de façon phénoménologique, selon trois catégories en les dissociant pour conduire à une référence en trièdre, utilisée de fait dans les schématisations procédurales. Imaginons un trépied de géomètre qui se regroupe en un axe et qui peut se déplier orthogonalement. La partie des intentions en projection avant, comme associée à 《des principes et des fonctions » peut être matérialisée par un axe vertical, rejoignant l'image platonicienne des idées générales. Quant au retour des aléas et des écarts, induit par la plongée des idées dans la complexité du réel et de l'opérationnel, c'est le domaine de la confrontation avec les dispersions des « objets et constituants » existants et des incertitudes liées à leur cycle de 


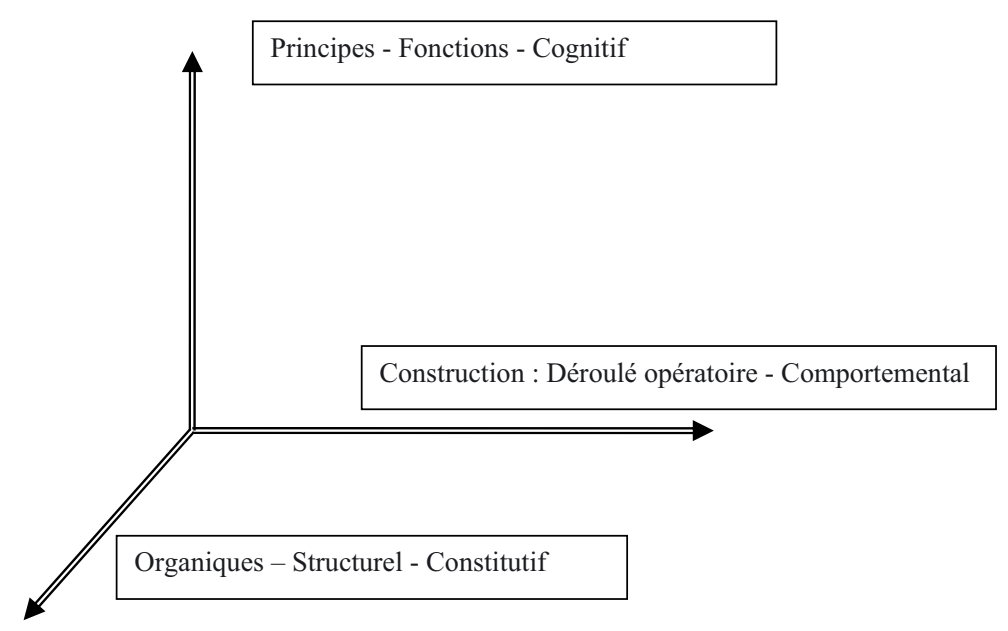

Fig. 3. Trièdre de positionnement : organiques/successivité/fonctions.

vie. Ceci est porté par l'axe horizontal normal à la feuille, descriptif des structures et constitutions, tandis que l'enchaînement des processus dans le « déroulé » opératoire s'inscrit, comme en mécanique, selon l'axe du temps ou plus exactement de la successivité [62] des séquences de la construction : comportementale et constitutive, mais aussi cognitive.

Ainsi exprimé, ceci peut apparaître simpliste, car occulté par son évidence. Mais ce trièdre (constitutif/comportemental/cognitif) est souvent utilisé, partiellement en bidimensionnel comme cadre de présentation $[13,44]$ de la plupart des schémas et organigrammes [63] systémiques. Quelques notes pour situer son emploi par rapport aux conditions initiales des figures précédentes (intentions, connaissances, antériorités, moyens) :

- « où » je vais : visées initiales (connaissances, intentions) ;

- «d'où » je viens : possibilités initiales (antériorités, moyens) ;

- « avec qui et avec quoi, par où » flux : (énergies, produits, acteurs, signaux) ;

- « comment $»$ : modalités constructives (pédagogies : technologie et pratique des professionnels et ingénieurs), comportementales (par analogie [64]), de conduite [49], de connaissance...

\subsection{Déroulé opératoire}

\section{Chaînage des processus par jalons, étapes et phases}

Cette désignation parlante (traduction de flow-chart) était plutôt utilisée pour ce que l'on nomme actuellement processus de fabrication. Passer de l'idée à l'objet, revient à construire cet objet à partir de fonctions d'usage désignées comme fonctions principales. Elles s'appuient sur les fonctions techniques, qui, regroupées, sont porteuses pragmatiquement de l'ensemble des flux en terme de performance, coût et délais ( triangle du projet ») et qui, sous l'angle où nous nous situons sont parfois antinomiques et doivent faire l'objet d'un compromis ou d'un arbitrage dans un projet en s'appuyant sur des modèles éprouvés. Ceci parfois occulté dans les chaînes de valeur économiques, constitue une source d'insatisfaction, voire d'échec.

L'abord direct par un schéma global tel celui de l'AUM sur le site de l'AFM, sous l'angle de la sensibilisation aux interactions scientifiques, ne peut faire apparaître ces contraintes d'articulation [65] de l'activité économique immédiate, auxquelles est soumise la mécanique dans son application industrielle. La logique de la conception transcrite dans cette séquence [66], comme série linéaire ordonnée de termes est insuffisante pour assurer la validité et doit s'inscrire dans une séquence avec des composantes itératives.

Cette démarche peut s'ajuster par une connaissance approchée [67] en amont, représentative des modèles et des connaissances, puis, en aval, descriptive de l'environnement. Dans le courant du déroulement, les vérifications des tolérances des procédés et les validations incorporent les incertitudes plausibles des processus. De même les qualifications ou homologations, s'appuient sur des marges supplémentaires, à reformuler par l'ingénierie système, pour le cas des logiciels [43], centrés sur la validation en situation opérationnelle.

En outre, la bonne fin repose sur la bonne orientation et la stabilité des opérations en œuvre, déjà par le rassemblement initial, avant le cursus, de l'équivalent des données et résultats décrits précédemment par l'association des conditions initiales (connaissances, intentions, antériorités, moyens). Ensuite, les éléments proactifs (selon le vocable de l'ingénierie des systèmes) ou projectifs, voire prévisionnels selon les disciplines, permettent l'affichage des objectifs retenus par les spécifications. L'obtention est contrôlée et stabilisée par des rétroactions au cours du déroulement. Enfin certains points sont peu accessibles, tels les composants sur étagères, l'usage des règles de confidentialité de protection, voire du fait du mutisme linguistique, de l'oubli malencontreux, de la déformation par transmission et perte de sens [42].

On identifie sur la figure 4 des règles simples, voire quelques prescriptions de management [63]. 


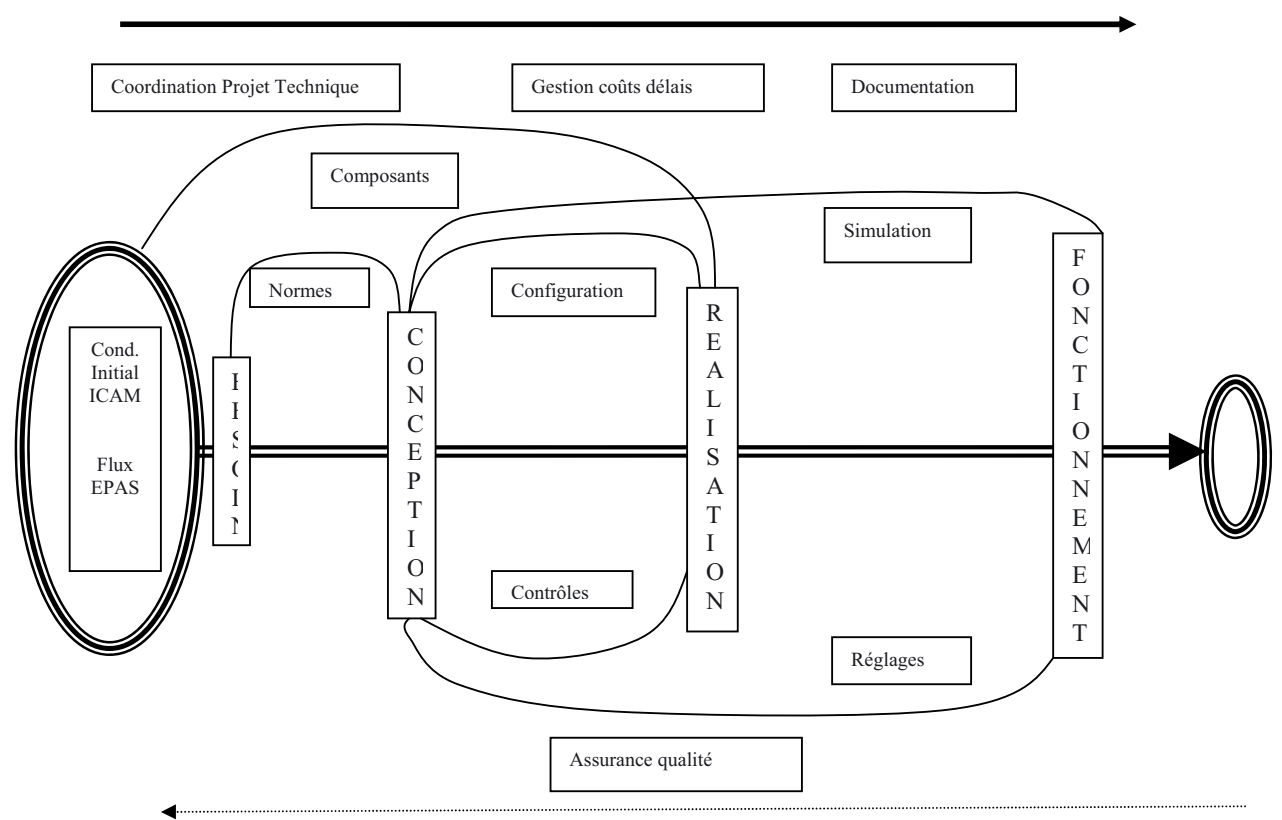

Fig. 4. Schéma de déroulé opératoire.

C'est ce qui est à l'origine, en bonne part, du détail des règles de management [43], souvent volumineuses comme dans les missions Apollo et suivantes [30], audelà de la complexité conceptuelle des produits modernes. Ce déroulé opératoire se fait, pour un développement complet, par un enchaînement de phases (FDQP : faisabilité, développement, qualification, production, simplifiant l'ancienne séquence ([18], démarrage, organisation, construction provisoire, construction proprement dite, prototype, essai du système) et plus pratique que celle de l'IS ([17] « definition, development, deployment »). Chacune des phases est constituée d'étapes associant des documents. La séquence que nous avons schématisée peut être désignée par le sigle « BCRF » [58], dont la terminologie est à prendre ici au sens mnémotechnique et pragmatique plus qu'au pied de la lettre : besoin, conception, réalisation et fonctionnement.

Pour les projets, des normes définissent les termes effectivement employés. Néanmoins, on peut noter les articles essentiels inclus dans la documentation type associée, derrière les présents vocables, utilisés de fait par de petites entités :

- étape B : Cahier des charges $=\mathrm{CF}=$ Besoin et contexte. Fonctions techniques et contraintes avec critères (spécification technique ST approuvée par les deux parties pour un travail industriel, ce qui nécessite une pré-étude relativement substantielle : cf. Fig. 6);

- étape C : Dossier de conception et définition DD = concept objet et $s / \Sigma$ (sous-système), justification des dimensionnements et choix, définition organique et disponibilité ;

- étape R : Dossier de Réalisation DR = séquences industrielles par éléments et d'ensemble (approvisionnement matières et produits, réalisation pièces, assemblage mécanique, intégration fonctionnelle);
- étape F : Fonctionnement (épreuve, suivi opérationnel) : plan d'essai PE et rapport d'essais RE : plan, conduite des essais, résultats, interprétation/exploitation, transfert.

Tous ces éléments sont justifiés par les besoins de conduite de projet, à savoir coordonner, réutiliser et transmettre. Ceux-ci sont à traduire dans les contextes indiqués précédemment, plus orientés vers la construction de la connaissance. Ils sont plus diversifiés et spécialisés pour une construction intégrée dans une démarche systémique, applicable à des projets industriels (cf. les spécifications de management BNAé, CNES, ECSS...).

\subsection{Situation de développement}

\section{Structures et indicatifs élémentaires et globaux}

On peut décrire quelques limites des différents types de cycle de développement (conception simultanée reprenant l'idée du « concurrent engineering », cycle en V fortement médiatisé...) sous la forme optimale du cycle en cascade des grands projets spatiaux.

L'intérêt de ce cycle apparait sur l'étude NASA cidessous, rarement mise en exergue, adaptée selon les concepts du présent article et montrant l'existence d'une situation optimale entre l'enchaînement en série des travaux et la conduite d'actions engagées simultanément.

On ajoute à ce constat empirique, le rappel de l'usage pragmatique, des préceptes cartésiens et de leur transposition systémique, schématisé à partir des propositions de Le Moigne [56] :

- Principes cartésiens d'aspect analytique, basés sur des chaînes de raisonnement :

- évidence d'objets distincts ; 


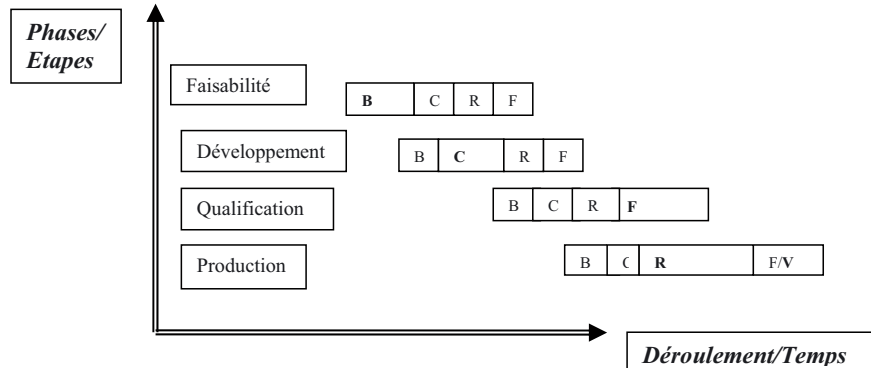

Fig. 5. Schématisation phases en cascade : FDQP.

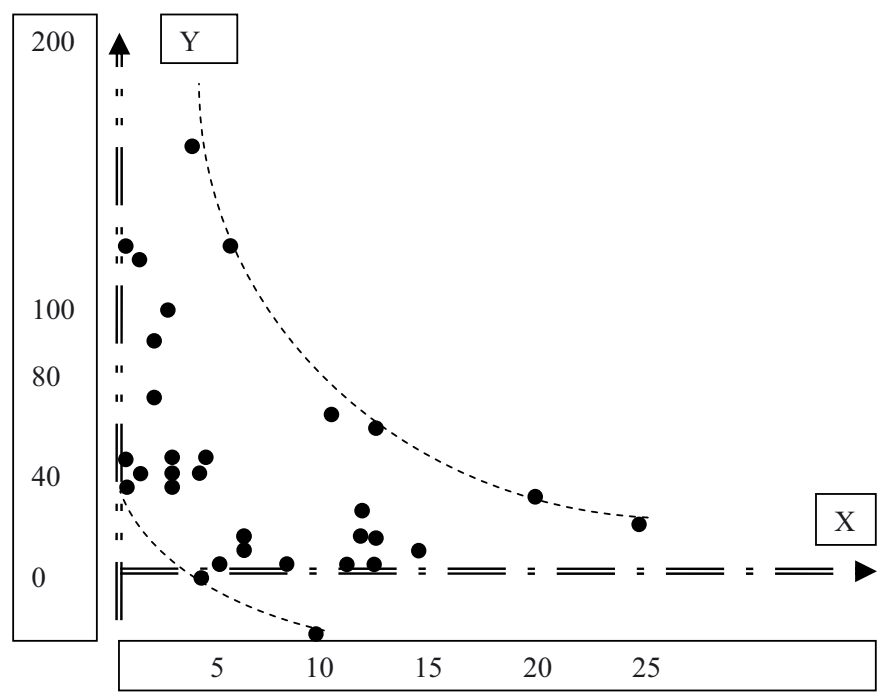

Fig. 6. Adaptation de NASA HQ W. Gruhl 1985 in SP NASA 6017 System engineering : Y = Dépassement final/ Engagement en phase $D-X=\%$ Coût faisabilité/Coût développement.

- réduction des objets manipulés ;

- liaison ordonnée du simple vers le composécausalité;

- vérification par un dénombrement exhaustif;

- Principes systémiques en synthèse d'aspects comportementaux, par combinaisons de notions statistiques, thermodynamiques, structuralistes, et cybernétiques :

- pertinence d'objets définis par rapport aux intentions du modélisateur ;

- globalisme de la relation fonctionnelle avec son environnement;

- finalisme descriptif puis constructif de l'objet par rapport à l'objectif;

- identification d'agrégats par et dans une sélection représentative.

\subsection{Transition modèle vers application}

\section{Représentation des formalisations industrielles}

Les analogies présentées ci-avant sont particularisées selon le poids industriel ou exploratoire tel que les schémas reliant la mécanique aux différents domaines de l'activité économique. Les paragraphes précédents se présentent comme une contribution à la description d'actions coopératives entre entités et acteurs, correspondant au début du développement des outils informatiques et bureautiques.

Néanmoins n'est abordé que l'enchaînement des démarches, sans mentionner toute la pratique associée à la mise en œuvre des dessins, calculs, intuitions et décisions en partie rédigées, opérations d'assemblage et d'intégration mécanique et fonctionnelle, programmées et enregistrées, préalables aux expérimentations de vérifications et d'explorations des domaines. Les pratiques industrielles ou de recherche font l'objet de formalisation que chacun perfectionne, en visant la conservation de l'acquis, avec les moyens nouveaux.

\section{Quelques pratiques actuelles}

On constate la conjonction [68] « d'une logique de l'offre [... et] d'une logique de la demande et également une prise en compte accrue de l'environnement industriel et de son évolution ». En outre « le besoin de formalisation de la connaissance », apparaît pour maîtriser la compréhension devant l'explosion combinatoire associée à l'évolution des outils diversifiés et souvent encapsulés et assurer les vérifications. En effet, l'enchaînement des étapes consiste à se positionner sur un point initial (point ou domaine nominal, limite, extrême) et à explorer par approximations successives (calcul, définition, réalisation, contrôle, essais divers) le domaine de validité du concept de l'objet et des modèles applicables associés. La théorie permet de se situer plus rapidement dans un contexte de décision et d'action. Pour un produit industriel, ceci s'applique plus particulièrement aux évolutions à partir des produits antérieurs. Sans forcer le lien avec les concepts descriptifs présentés ci-dessus, on peut indiquer quelques pratiques récentes avec les articulations propres aux spécificités des domaines et des acteurs.

\subsection{Structures et mécanismes}

Combinaison de tracé, de dessin, de standard et de calcul de dimensionnement

Les thèmes habituels en construction pour les mécaniciens [59] sont le point de rencontre des démarches précédentes associées aux techniques et pratiques du dessin, réadaptées aux logiciels actuels.

Avec l'acquis de la pratique, la mise en place géométrique en relation avec les appuis et les axes de chargement se fait assez naturellement avec l'expérience, en suivant les lignes de flux supposés et les axes de référence choisis intuitivement. Le dimensionnement mécanique intervient en parallèle, et spécialement, au vu des formes particulières induites par l'assemblage imaginé et la fabrication disponible. Le dessin est plus immédiatement porteur du concept en même temps que de la définition répondant aux spécifications précises. La vérification des marges [69] se fait par rapport aux facteurs de sécurité 
associés au domaine d'emploi dans des points particuliers et maintenant par une vision globale avec les outils de calcul en 3D, des contraintes et déformations, disponibles sur ordinateur de bureau. Du point de vue des interfaces cinématiques ou quasi-statiques, avec des conditions particulières de lubrification souvent associées à celles du fonctionnement prototype en milieu extrême, il est nécessaire de procéder à des calculs de génération thermique et de vérifier les conditions de frottement limite. On doit noter qu'il est préférable d'éviter les revêtements et traitements de surface sur les matériels en petite série et de privilégier, quand c'est possible, des matériaux massifs compatibles avec cette fonction technique de contact.

On entre alors dans une démarche pluridisciplinaire, du fait des spécialisations industrielles et des domaines de recherche à travers le mélange de la technologie et de la tribologie, qui conduit à examiner :

- les modes d'assemblage dans l'objet;

- les conditions de positionnement dans l'installation de traitement ;

- les tolérances et rugosités des substrats pour l'adhésion et l'accrochage;

- l'adéquation des conditions thermiques du traitement de surface avec les états de traitement métallurgique et les contraintes résiduelles du substrat;

- la compatibilité physico-chimique des interfaces avec le milieu environnant.

Malgré les succès reconnus en mécanique, on ne résiste pas à évoquer une petite histoire symptomatique à l'égard de la mécanique technologique. Lors d'une réunion générale préparant la constitution du Groupement de recherche sur les roulements, Jacques Cliton l'enseignant de l'ENSAM en mécanique vibratoire a rappelé une phrase ouvrant le cours de licence à la faculté de Paris avant-guerre : « nous ne parlerons pas des roulements, cela ne sert que dans la pratique $»$.

Maintenant, la démarche de construction d'un mécanisme ou d'une structure peut consister après une phase de prédimensionnement par équations analytiques (Mathematica, Matlab, TkSolver...), à réaliser au plus tôt un tracé en CAO des objets. Ce tracé permet, avec l'expérience, de mieux identifier les contraintes, les fonctions techniques associées et de réaliser les calculs de prédimensionnement de l'ensemble des caractéristiques (masses, inerties, surfaces données en direct par la $\mathrm{CAO}$, contraintes mécaniques obtenues rapidement avec éléments-finis intégrés), avant les calculs plus complexes en itération avec la CAO et un code aux élémentsfinis (Nastran, Accord, César..., en thermique et dynamique et avec des lois de comportement linéaires ou nonlinéaires). Ce tracé partiellement dimensionné conduit à un plan avant-projet qui est un support à la réflexion et à l'identification des fonctions et contraintes dites secondaires, mais souvent essentielles (étanchéité, paliers...). Le modèle analytique peut éventuellement être mis à jour en parallèle, car il fait gagner du temps en utilisation comme outil de prédiction de l'influence de la variation des paramètres, ce qui limite le nombre de calculs en éléments finis toujours plus longs à effectuer et à exploiter. La CAO utilisée tôt facilite également l'examen des moyens de réalisation qui seront employés : à partir d'un plan, même provisoire, il est possible de valider le lancement des approvisionnements et de la réalisation. On doit situer tôt les problèmes associés aux dimensions, grandes ou au contraire très petites, et en particulier quant à la précision.

\section{2 Écoulements}

Itérations de I'unidimensionnel au tridimensionnel

Du fait de la priorité historique en R\&T, du fonctionnel de la «propulsion par fusées [due]... à l'apparente simplicité du problème [qui] dissimule en fait de très sérieuses difficultés [70] » par rapport à la mécanique structurelle on cite deux exemples.

Tout d'abord un travail de modélisation essentiellement unidimensionnel du comportement insatisfaisant de l'alimentation de l'étage supérieur, au lancement 510 d'Ariane 5, la note technique [71] concernant le transitoire d'allumage est un exemple de travail de simulation représentatif d'une situation, selon le processus d'analyse phénoménologique de Darrozes [72] et l'utilisation de stratégie de résolution des équations adaptées au problème particulier. En ce qui concerne les pompes, la plateforme du LEMFI/LTCE, piloté par Rey [73], permet, en partant d'un cahier des charges, de définir rapidement avec un logiciel monodimensionnel un premier tracé de la coupe méridienne d'une pompe (centrifuge ou axiale).

Ensuite après une ébauche de la constitution circonférentielle (nombre d'aubages, angle d'attaque...), on peut procéder à une vérification de l'écoulement avec un code 3D (type Fluent) et, après analyse de points particuliers, à un dessin technologique 3D de l'ensemble de la partie tournante de la pompe, réalisable par stéréolithographie en quelques jours. Ceci est une application pour concevoir la géométrie d'un objet technique identifié. Il convient d'en faire le dimensionnement aux charges mécaniques et thermiques et de l'insérer technologiquement dans un équipement soumis aux contraintes et sollicitations du milieu extérieur, constitué à la fois par sa propre veine fluide et ses appuis mécaniques.

\subsection{Actionneur électromagnétique rectiligne}

\section{Interaction des technologies et paramètres mécaniques et électriques}

Un cas d'association à la mécanique des techniques et outils de l'électrotechnique et de l'automatique est matérialisé par la réalisation d'une pompe embarquée dont le principe utilise un actionneur à mouvement rectiligne, par commande électromagnétique avec réluctance variable. Celui-ci répond en partie au défi posé par 
Rocard [74] sur le pendule entretenu par un courant alternatif, grâce aux nouveaux matériaux et aux capacités des composants électroniques et à la versatilité ouverte par les outils logiciels. La conception et la définition des éléments mécaniques, tant structuraux que fluides, ont été entreprises selon les indications données précédemment. Quant à la mise au point de systèmes de commande tant en tension/courant qu'en position, décrite par Vannier [75], elle repose sur l'emploi d'outils de calcul formel à partir de schématisation de diagramme de commande permettant la mise en ouvre de cartes temps réel assurant une commande par MLI (modulation de largeur d'impulsion - désigné antérieurement par le sigle anglais PWM) des courants d'alimentation. Cette démarche a permis de réaliser un prototype et de vérifier le fonctionnement avec différentes modalités de commande de position s'appuyant sur l'utilisation des équations d'état complétée par un correcteur adapté aux non-linéarités en dehors du point nominal de modélisation. En outre le système repose sur un capteur de position intégré apte à fonctionner selon les matériaux des contrefaces en courant de Foucault ou en réluctance variable. Ce dispositif en cours d'évolution se prête à la commande précise de position.

\subsection{Installations expérimentales}

\section{Management de la diversité et pilotage de la pluridisciplinarité}

Au-delà de l'imbrication des outils décrite ci-dessus, on peut donner comme exemple actuel d'association de démarches inspirées par les principes évoqués, celles du GdR dirigée par Michel Dudeck [28] utilisant le banc Pivoine à Orléans. Il en est de même avec le banc Balafre cofinancé par des institutions et des industriels, dont le management est assuré par le LMS Poitiers au bénéfice des contributeurs utilisateurs. Ces acteurs ou parties prenantes sont le CNES, la SNECMA Vernon, l'EDF, Alstom, le CNRS, la Région Poitou-Charentes, le Conseil général de la Vienne, l'université de Poitiers. Tout ceci conduit à chercher :

- à la fois la simplicité de la conduite des tâches;

- la prise en compte des ordres de grandeur pour le dessin de mise en place ou d'architecture;

- les vérifications plus détaillées pour les points sensibles appelés échantillonnage sur les dimensionnements des structures aéronautiques entre les deux guerres;

- à utiliser des logiciels de calcul formel ;

- à classer les activités pour un meilleur pilotage des commanditaires par l'utilisation actuelle de la maturité de sujets par la démarche des classes dites de maturité technologique (TRL pour « Technology readiness level $\gg$ de la Nasa).

Mais ceci ne suffit pas. Il faut ajouter l'aptitude professionnelle des enseignants-chercheurs quant à l'observation des personnes, la conduite prudente de l'évolution des sujets, associée à une vision lointaine des problèmes tant dans le concept que dans le détail de la réalisation. On doit ajouter une variété de caractères, comme par exemple celui de René Pellat gentiment bien défini par Curien « compétent et pas coincé ». Quant aux vivants, pour mettre en exergue l'importance des personnalités, on ne peut que citer la culture de leur domaine de physiciens, de tribologues, de mécaniciens des fluides et thermodynamiciens, de thermiciens, de spécialistes de la combustion et des plasmas, de l'informatique et de la fiabilité. Et, mécaniciens, nous devons ajouter pour les dernières activités citées, les électrotechniciens, électroniciens et automaticiens. Les échanges et les observations réciproques ont été source d'innovation et de vérification.

\section{Conclusions}

Pour faire partager une expérience de pratique de la mécanique, nous présentons un point de vue sur la construction d'objets techniques. Les descriptions précédentes impliquent des itérations autour de l'objet principal, du fait de l'intrication des démarches et surtout de l'optimisation, par exemple des masses, qui deviennent un critère économique. Ici on se situe en plein dans la démarche « du modèle à l'application », où, selon un adage, on construit mieux ce que l'on sait tracer et calculer dirait-on maintenant, en passant par une prise en compte des ordres de grandeur [72], voire la linéarisation, mais aussi la similitude [76].

Pour calculer, on constate qu'il faut imaginer et tracer au préalable des éléments d'objet à partir des fonctions, soit par analogie, soit en partant de procédés de construction innovants ou éprouvés. De la même manière on mesure mieux ce que l'on a d'abord défini. On peut imaginer que les objets sont suffisamment définis par des documents assurant des relations efficaces à partir de données cohérentes, en vue de réaliser des ensembles homogènes, fournissant des services adéquats. Mais l'expérience montre que, même si tout ceci est une référence indispensable, il est nécessaire de réajuster les objectifs et de corriger les écarts en tenant compte des dispersions [77] en permanence au long du cycle de développement.

Cette démarche s'articule autour des fonctions techniques, ici mécaniques, d'un objet industriel, selon une vision systémique, utilisant des «Flux» (énergie, produits, acteurs, signaux) partant de conditions initiales (intentions, connaissances, antériorités, moyens). Cette séquence est ajustée par des « bouclages projectifs et rétroactifs », immédiats, ou positionnés en fin d'étape, de phase ou de programme. Cette représentation permet également la validation de la démarche de construction et l'adaptation des connaissances et des technologies à des environnements différents, selon la fameuse expression des retombées.

Le déroulé opératoire de cette séquence, traduisant les besoins en idées puis en fonctions et enfin en éléments concrets, comporte la conception et définition pour la 
réalisation d'un objet. Celui-ci pour être apte à l'usage doit passer par la vérification de sa constitution en correspondance avec le cahier des charges, le contrôle de la réalisation et la validation du fonctionnement avec marges avant transfert à l'utilisateur

Cette démarche de construction présente dans une schématisation géométrique, des aspects descendants et ascendants, le long de l'arborescence des objets ou systèmes et de leurs constituants. On ne doit pas oublier les connections horizontales ou transversales dans un tel réseau pour comparer des éléments similaires ou associer les connaissances de différents domaines ou entités. Pour construire matériellement les éléments, en s'assurant de leur dimensionnement fonctionnel et structural en conception, on se situe dans des plages acceptables quant aux aspects techniques (performances, sûreté, innocuité...) et économiques (coût, disponibilité,...) pour un bon comportement des matériaux et composants. On utilise les propriétés issues de la séquence : élaboration des bruts, usinage des demi-produits, enroulement des composites, traitements divers, assemblages des composants, caractérisation des comportements.

Ce mode d'exploration est à élargir par celui des conduites d'essais [49] avec toute la statistique associée et, au-delà, par les démarches scientifiques de la connaissance, génératrices de meilleure maîtrise de la compréhension et de la mémorisation, car « la connectivité des deux domaines (scientifique et technologique) est si étroite que chacun se nourrit de l'autre et ne progresse qu'en s'appuyant sur l'autre » [78].

Il nous semble que le concours des différents acteurs de ces processus pourra contribuer à transposer le point de vue synthétique, mais partiel, exprimé par cet article et à l'utiliser selon les spécificités des équipes institutionnelles et industrielles. En effet la surabondance d'exigences peut nuire à la créativité tant pour l'innovation que pour la découverte. Pour constituer un espace de travail, l'échange est nécessaire avec d'autres acteurs, comme ceux, comme Klein pour qui « l'activité scientifique a pour but de nous rendre le monde intelligible, de créer des concepts et d'en tester la pertinence $»[79]$.

Cependant devant l'accroissement des contraintes et de la complexité, la connaissance scientifique tout autant que sa mise en forme adéquate aux processus de construction et d'utilisation en général, ne peuvent se développer sans la convergence de concours industriels et institutionnel, dans le cadre de projets d'application. La réussite de la fusée Ariane a reposé et repose sur ceux-ci à travers la participation essentielle des acteurs décisionnaires, mais aussi sur l'implication passionnée des ingénieurs et des scientifiques spécialistes des sciences mécaniques et physiques. La relève des versions de lanceurs actuellement en usage suscite des interrogations [80]. Souhaitons que cette source ne se tarisse en entraînant des répercussions au-delà des seules applications à terme, commerciales ou scientifiques de l'univers, elles aussi tributaires d'une coopération multidisciplinaire dans un contexte actif et concret, une des voies, nécessaire au progrès de la connaissance pour la construction mécanique.

\section{Références}

[1] F. D'Allest, Why is the most widely used launcher in the world European, ESA-SP-387

[2] W.J. Larson, J.R. Wertz, Space mission, analysis and design, Kluwer, 1992

[3] CNES L'ambition technologique : naissance d'Ariane, Inst. d'histoire de l'industrie, 1995

[4] H. Morand, R. Ohayon, Interactions fluides-structure, Masson, 1992

[5] A. Beaurain, A. Souchier, La préparation des nouvelles générations de moteurs fusées à ergols liquides à SNECMA, Revue Sci. Tech. de la défense 60 (2003)

[6] J.M. Fouet, Connaissances et savoir-faire en entreprise, Hermès, 1997

[7] J.M. Juran, Quality control handbook, Complex system section 44 (1974)

[8] C. Talbot, J.M. Astorg, J. Berenbach, E. Louaas, M. Illig, C. Bonnal, Possible new expandable launchers for Europe in the future, IAC-07-D2.4.04 58th IA congress Hyderabad 24-28 September 2007

[9] R. Pellat, De la mécanique à la propulsion - un enjeu pour la recherche, Année de la Mécanique Groupe SNECMA, Unesco, 1988, p. 35

[10] D. Bellet, Mécanique Générale Cepadues, 1988, p. 355

[11] J. Bricmont, A. Sokal, Impostures intellectuelles, Odile Jacob, 1977

[12] J. Bouveresse, Prodiges et vertiges de l'analogie, Le Seuil, 1999

[13] J.M. Albertini, L'économie en 200 schémas, Les éditions de l'atelier, 1994

[14] H. Le Chatelier, Science et Industrie, Flammarion, 1925, réédité CTHS 2001

[15] J.J. Gagnepain, Préface de « Un siècle de recherche technologique », CNRS, 2000

[16] J.M. Albertini, A. Silem, Comprendre les théories économiques, Seuil, 2001

[17] Y. Deforge, Technologie et génétique de l'objet industriel, UTC

[18] H.H. Goode, Méthodes d'études des systèmes techniques complexes, Dunod, 1963

[19] A.P. Sage, Armstrong, Introductions to system engineering, Wiley, 2000

[20] P. Feller, F. Tourret, Ph. Schlienger, L'outil, $\varepsilon \mathrm{pA}$ Hachette, 2004

[21] J. Baudet, De l'outil à la machine; De la Machine au système, Vuibert, 2003

[22] S. Nora, A. Minc, Rapport sur l'information de la société, Seuil, 1978

[23] A. Boyer, Le tour de France d'un compagnon du devoir, Imp. du compagnonnage, 1975

[24] J. Pitrat, Métaconnaissance - futur de l'intelligence artificielle, Hermès, 1990

[25] A. Lichnerowicz, F. Perroux, C. Gadoffre, Séminaires interdisciplinaires, Analogie et connaissance, Maloine, 1980

[26] Th. de Montbrial, L'action et le système du monde, PUF, 2002

[27] B. Lussato, Introduction critique aux théories d'organisation, Dunod, 1972

[28] M. Dudeck, La propulsion électrique pour les missions spatiales, Lettre AAAF $2 \operatorname{Tr} / 2007$ 
[29] J. Frene, D. Nicolas, B. Degeurce, D. Berthé, M. Godet, Lubrification hydrodynamique, Paliers et Butées, Eyrolles, 1990

[30] Anonymous, NASA SP 6107, System engineering

[31] M.A. Paveau, G.E. Sarfati, Les grandes théories de la linguistique, A. Colin, 2003

[32] G. Guillaume, Langage et sciences du langage Nizet, 1964 (schémas précisées par E. Ortigues Le discours et le symbole 1962)

[33] G. Guillaume, R. Valin, Époques et niveaux temporels dans le système de conjugaison française, Presses universitaires Laval, 1955, p. 7

[34] F. Dugué, Conception et vérification des paramètres tribologiques, (in [50])

[35] G. Jeanblanc, Dispositif d'essais de roulements sous ultravide, La technologie spatiale française, CNES, 1971

[36] S. Potteck, La conception des grands systèmes 1999/2001, éditions du Schemectif

[37] C. Petitdemange, La maîtrise de la valeur, AFNOR Gestion, 1985

[38] L. Géminard, F. Gros la Faige, Construction mécanique, Dunod, 1966

[39] L. Géminard, Logique et technologie, Dunod, 1970

[40] R. Tassinari, La maîtrise des coûts industriels, E d'Organisation, 1981

[41] J.L. Fanchon, Guide des sciences et technologies industrielles, AFNOR, Nathan, 2008

[42] C. Morel, Les décisions absurdes, Gallimard, 2003

[43] J.P. Meinadier, Intégration des systèmes : QSJ, 1997, puis Hermès, 1998, 2002

[44] L. von Bertalanffy, Théorie générale des systèmes, Dunod, 1973

[45] J. Lesourne, Les systèmes du destin, Dalloz économie, 1976

[46] A. Lebeau, La navette spatiale : autopsie d'une faute stratégique, Futuribles, 2006

[47] P. Watzlawick, Une logique de la communication, Seuil, 1979

[48] Confederation of European Environmental Engineering Societies, Paris : ASTE, Règlements REACH et RoHs

[49] P. de Larminat et Y. Thomas, Automatique des systèmes linéaires, Flammarion 1977

[50] G. Jeanblanc, G. Inglebert, Roulements et Tribologie, 1994/1999, SIRPE 2001, GST/AFM tribologie édités maintenant par les PPU, Romandes

[51] G. Jeanblanc, Logique de conception appliqué à des éléments de machines, Enam 0015, 1990, Structures techniques, Supélec 0742/2002

[52] D.R. Earles, MF Edding Reliability physics (The physics of failure), Symposium, 1966

[53] COTS : Component on the shelves = matériels prédéfinis, existants sur étagères

[54] J.C. Ligeron, Le management des grands programmes, Eyrolles, 1988

[55] Strategor : Stratégie, structure, décision, identité, InterEditions, Groupe HEC, 1994
[56] J.-L. Le Moigne, La Théorie du système général, PUF, 1977-1984

[57] J.L. Ermine, Les systèmes de connaissance, 1996

[58] G. Jeanblanc, P. Galley, F. Dugué, Mémoire technique en mécanique, GAMI, 11, 1988

[59] S. Tichkiewitch, Conception de produits mécaniques, Hermès, 1998

[60] E.A. Orady, I. Shareef, Expert system design philosophy for application of simultaneous engineering in industry, in Design for manufacturability, 1993

[61] Space mission, analysis and design, W.J. Larson and J.R. Wertz, Kluwer, 1992

[62] F. de Saussure, Cours de linguistique générale, Payot, 1989

[63] Organigrammes des institutions françaises, collection U, A. Colin, Préface G. Vedel, 1971

[64] J. Lemaitre, J.L. Chaboche, Mécanique des milieux solides, Dunod, 1988

[65] P. Vendryes, Vers la théorie de l'homme, PUF, 1973

[66] Le Robert, Dictionnaire historique de la langue française, 1998, p. 3471

[67] G. Bachelard, Essai sur la connaissance approchée, J. Vrin, 1987

[68] Y. d'Escatha, Préface « De l'idée au produit », P. Maitre, J.-D. Miquel, Eyrolles, 1992

[69] V. Verderaime, Total systems design analysis of high performance, NASA, 1993

[70] M. Roy, préface p. VIII de M. Barrère et al., La propulsion par fusées, Dunod, 1957

[71] G. Albano, B. Legrand, P. Vuillermoz, NT n ${ }^{\circ} 149$ CNES, Modélisation du transitoire d'allumage d'un moteur à ergols liquides, 2003

[72] J.S. Darrozes, A. Monavon, Analyse phénoménologique des écoulements, UMPC, 2002

[73] R. Rey, R. Noguera, M. Asuaje, Computer aided design and optimization of centrifugal pumps, Proc. Imec E, J. Power Energy 219 (2004) 11

[74] Y. Rocard, Dynamique des vibrations, Masson, 19431949, Paragraphe 162

[75] J.-Cl. Vannier, A. Arzandé, H. Siguerdidjane, P. Vidal, F. Dugué, Control of a new hydrazine pump generation in propulsion system, 2005

[76] L.I. Sedov, Similarity and dimensional methods in Mechanics, Infosearch, London, 1959

[77] R.S. Ryan, J.S. Townsend, NASA MSFC, Application of probabilistic analysis/design and methods in space programs: the approaches, the status, and the need, AIAA 93-1381

[78] H. Curien, Découverte et innovation, Le Monde, 27 octobre 1999 , p. 17

[79] E. Klein, L'avenir de la recherche scientifique, Études, juin 2008

[80] F. D'Allest, La fusée Ariane menacée de disparition, Le Monde, 6 août 2008, p. 14 